\title{
OPTIMIZATION OF THICK FILM RESISTORS FOR LOW DRIFT
}

\author{
GUNNAR BJÖRKLUND and LARS LINDE \\ Rifa AB, Spånga, Sweden
}

(Received June 26, 1979)

\begin{abstract}
In order to minimize drift in a thick film resistor both printing and trimming parameters have to be optimized carefully

With the aid of a linear theoretical model it is shown how the resistance drift in the bulk transition zone and terminals of the resistor can be calculated for both trimmed and untrimmed resistors.

The theoretical results are used for optimization of laser trimmed high ohmic resistors. The experimental results are in good agreement with the theoretical predictions and confirm that thick film resistors can be optimized for low drift.
\end{abstract}

\section{INTRODUCTION}

Cermet thick film resistors are manufactured in a screen printing process and fired at a high temperature $\left(800-900^{\circ} \mathrm{C}\right)$. As described by Coleman, ${ }^{1}$ the electrical properties of the resistors are determined by conducting particles embedded in a glass matrix. Since the printing and firing processes alone are not sufficient to guarantee low tolerances in resistor values, trimming is necessary.

High speed industrial trimming of thick film resistors is to-day performed by Q-switched YAG-lasers. Normal pulse-rates are about $5000 \mathrm{~s}^{-1}$ Each pulse will cause the resistor material to evaporate, leaving a kerf with c:a $40 \mu \mathrm{m}$ width in the resistor bulk. The elevated temperature during the trimming operation will increase the risk of microcracks in the resistor material. ${ }^{2,3,4}$ This might cause drift in the trimmed resistor (both in $R$ and $\sigma$ values) according to Figure 1.

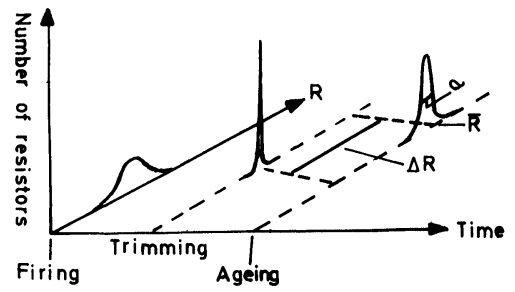

FIGURE 1 Increase in resistance values after trimming and ageing.
For high stability resistors, i.e. resistors with expected total drift less than $0.5 \%$ in a $70^{\circ} \mathrm{C}$ ambient, special care has to be taken in manufacturing. The aim of this paper is therefore to analyse the relative importance of the sources for drift in resistors of BiRu-type, together with a set of formulae which should serve as an aid for judgement of acceptable types of kerfs and degrees of trimming in order to optimize long term stability.

\section{THEORY}

The high ohmic thick film resistor in its "as fired" state consists of three zones (see Figure 2a) namely the terminal, the bulk and the transition zone.

The total resistance $R$ of the resistor can therefore be written as a sum of three terms:

$$
R=R_{\mathrm{bulk}}+R_{\text {trans }}+R_{\text {term }} ;
$$

Here $R_{\mathrm{bulk}}$ is the bulk resistance, $R_{\mathrm{trans}}$ the

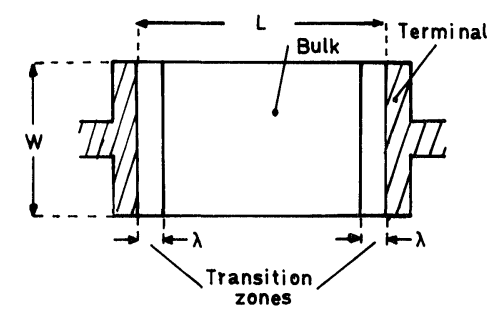

FIGURE 2a Schematic figure of the thick film resistor. 


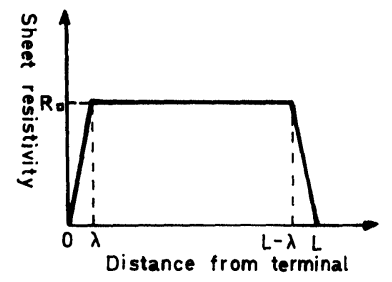

FIGURE 2b Linearized model of the sheet resistivity for an untrimmed resistor.

resistance of the transition zones, and $R_{\text {term }}$ the terminal resistance.

For a high ohmic resistor, $R_{\text {term }}$ can be neglected and we can therefore design the linearized model in Figure $2 \mathrm{~b}$ for the sheet resistivity in an untrimmed thick film resistor. $R_{\square}$ is the effective sheet resistivity of the bulk, and $\lambda$ is the effective length of the transition zone. Normally $\lambda$ can be considered as small compared to $L$ (of the order of $0.1 \mathrm{~mm}$ or less for single fired resistors). ${ }^{5}$ It is practical to consider $L-\lambda$ as the effective length $L^{\prime}$ of the resistor and by integration of the curve in Figure $2 b$ we obtain

$$
R=R_{\square} \frac{L-\lambda}{W}=R_{\square} \frac{L^{\prime}}{W} ;
$$

For a film resistor under environmental stress ageing will occur. In particular, an eventual increase in $R_{\text {trans }}$ will cause $\lambda$ to diminish by an increment $-\Delta \lambda$ (which means that $L^{\prime}$ is increased by $\Delta \lambda$ ). Similarly a change in $R_{\mathrm{bulk}}$ is characterized by a drift in the $R_{\square}$ value by $\Delta R_{\square}$. By logarithmic differentiation of Eq. (2) we therefore get:

$$
\frac{\Delta R}{R}=\frac{\Delta R_{\square}}{R_{\square}}-\frac{\Delta \lambda}{L^{\prime}} ;
$$

By laser trimming a kerf is made in the resistor bulk. This means that the resistor value in Eq. (2) will increase by a factor $f$ :

$$
R^{\prime}=f R_{\square} \frac{L^{\prime}}{W} ;
$$

As we have earlier mentioned, a small drift due to microcracking normally takes place after trimming. The microcracks are mainly located at the kerf tip $^{3,4}$ and will therefore be equivalent to an increase in the $l$-value (see Figure 3 ) by a small quantity $\Delta l$. This causes an increase $\Delta R^{\prime}$ in the resistor value and after logarithmic differentiation of Eq. (4) we obtain:

$$
\frac{\Delta R^{\prime}}{R^{\prime}}=\left(-\frac{d f^{-1}}{d \frac{l}{W}}\right) f \frac{\Delta l}{W}+\frac{\Delta R_{\square}}{R_{\square}}-\frac{\Delta \lambda}{L^{\prime}} ;
$$

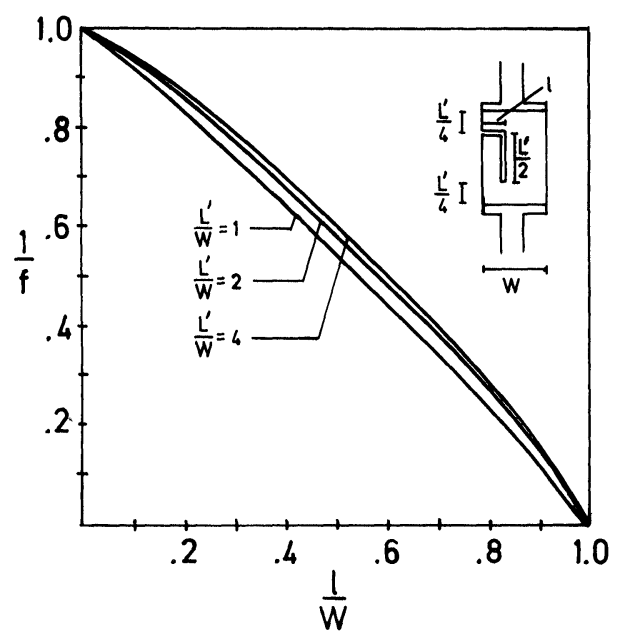

FIGURE 3a Numerically calculated $f^{-1}$ versus $l / W$ curves for $L$-cut resistors.

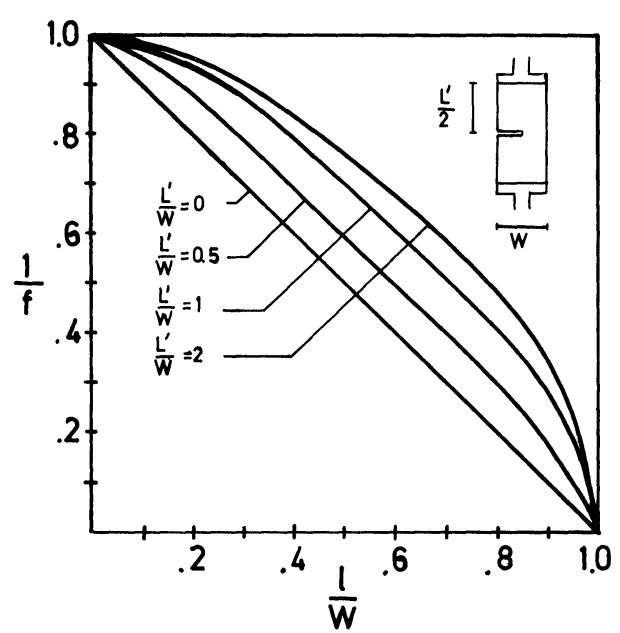

FIGURE $3 \mathrm{~b} \quad$ Numerically calculated $f^{-1}$ versus $l / W$ curves for $D$-cut resistors.

The value of $f^{-1}$ has been calculated numerically using the resistive network approach ${ }^{6,7}$ for both " $D$ " and " $L$ " cut. (See Figures $3 \mathrm{a}$ and $3 \mathrm{~b}$.) Eq. (5) allows for direct insertion of the $f^{-1}$ derivative. This is also practical from the point of view that this derivative is, to a good approximation, equal to -1 over the $f$ and $L / W$ ranges of most interest for the thickfilm designer. 


\section{DRIFT IN UNTRIMMED RESISTORS}

The linearized resistor model we have used in the preceding paragraph allows for separation between drift in $R_{\square}$ and $\lambda$ (or $L^{\prime}$ ) according to Eq. (3). We can see that if we plot $\Delta R / R$ as a function of $W /(L-\lambda)$ a straight line should result. In order to verify this experimentally, 3 series of 20 test substrates, containing 8 resistors with $W=2 \mathrm{~mm}$ and of varying $L$ values between 0.5 and $16 \mathrm{~mm}$, were printed. All three series were printed with 100 $\mathrm{kohm} / \square \mathrm{BiRu}$-type resistors of $12 \mu \mathrm{m}$ thickness after firing at $850^{\circ} \mathrm{C}$. The first group had the resistors printed on PdAg-terminals, the second group on $\mathrm{PtAu}$-terminals and the third group on fritless Au-terminals.

The relative drift was measured after $168 \mathrm{~h}$ and $500 \mathrm{~h}$ at $150^{\circ} \mathrm{C}$ and the mean values $\overline{\Delta R} / \bar{R}$ were plotted as a function of $W / L^{\prime}$ in Figure 4. By choosing $\lambda=0.1 \mathrm{~mm}$ for PdAg and $\lambda=0.045 \mathrm{~mm}$ for terminals containing $\mathrm{Au}$, the plot showed straight lines according to Figure 4. Eq (3) was thus confirmed and we were able to determine $\Delta R_{\square} / R_{\square}$ and $\Delta \lambda$ from the ordinate intercept and the derivative of these curves. For the reference curve we obtained $(\mathrm{PdAg}, 168 \mathrm{~h})$ : $\Delta \lambda=-410^{-4} \mathrm{~mm}, \Delta R_{\square} / R_{\square}=410^{-4}$. This means that a resistor of $1 \mathrm{~mm}$ length shows a total drift

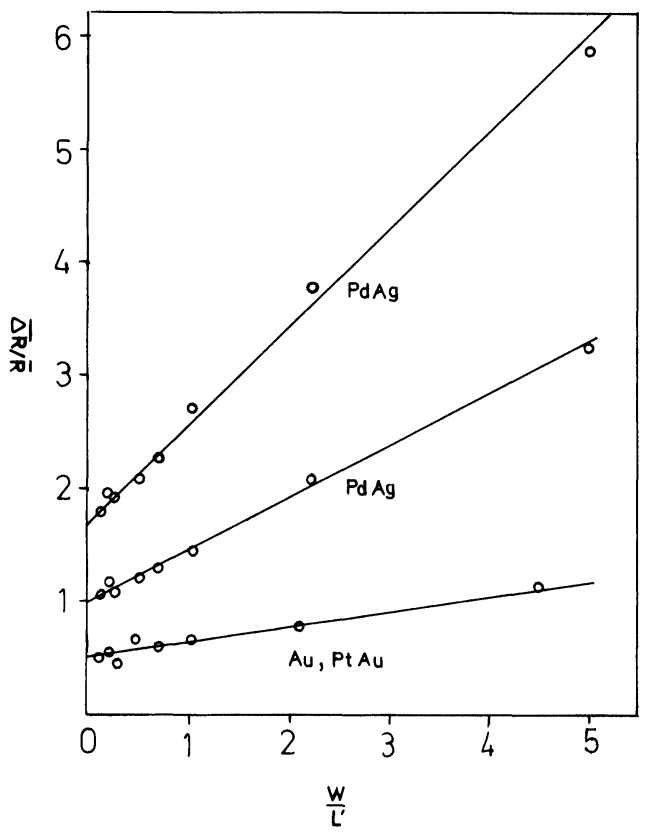

FIGURE 4 Normalized plot of $\overline{\Delta R} / \bar{R}$ at $150^{\circ} \mathrm{C}$. Upper curve: $500 \mathrm{~h}$ ageing. Middle (reference) curve and lower curve: $168 \mathrm{~h}$ ageing. which is equally distributed between bulk and transition zone

It is obvious from Figure 4 that the drift in resistors with $\mathrm{PdAg}$ terminals is considerably larger than for the two other types of terminals. Since all printing and firing parameters were identical, the most plausible explanation is diffusion of silver from the terminals into the resistor material. Another indication of this is the increased $\lambda$-value for the PdAg-group $(\lambda=0.1 \mathrm{~mm})$. This value is also in good agreement with experimental values found in both EMPA and microprobe voltage analysis on similar resistors. ${ }^{5}$

\section{DRIFT IN TRIMMED RESISTORS}

By rewriting Eq. (5) we obtain the following expression for $\Delta l$ :

$$
\Delta l=W\left(\frac{\Delta \lambda}{L^{\prime}}+\frac{\Delta R^{\prime}}{R^{\prime}}-\frac{\Delta R_{\square}}{R_{\square}}\right) f^{-1}\left(-\frac{d f^{-1}}{d \frac{l}{W}}\right)^{-1} ;
$$

We see that by using Figure $3 \mathrm{a}$ (for $L$-cut) and Figure $3 \mathrm{~b}$ (for $D$-cut), we are able to determine the last two factors in Eq. (6) for a given $f$ value and $L / W$ ratio. By using the experimentally found values of $\Delta R_{\square} / R_{\square}$, $\lambda$ and $\Delta \lambda$ from the preceding section, the remaining constants in Eq. (5) and Eq. (6) are known. Therefore only the $\Delta R^{\prime} / R^{\prime}$ value remains to be inserted in order to determine $\Delta l$.



FIGURE 5 Plot of $\overline{\Delta l}$ as a function of resistor geometry for $D$-cut (left curve) and $L$-cut (right curve). 
$\Delta R^{\prime} / R^{\prime}$ was determined by using experimentally measured drift in resistors (thermally aged during $168 \mathrm{~h}$ in $150^{\circ} \mathrm{C}$ ) with varying types of geometries and trim cuts. Two groups of resistors were investigated. The first group was trimmed with $L$-cut and the second one with $D$-cut. In total, 21 geometry-versuscut length combinations (each consisting of 20 resistors) were investigated. By inserting the experimentally found mean values $\Delta R^{\prime} / R^{\prime}$ in Eq. (6), the mean value $\overline{\Delta l}$ was calculated for each combination and plotted in Figure 5.

For $L$-cut resistors $\overline{\Delta l}$ is a weighted mean value for all microcracks penetrating from the walls of the trim kerf into the narrow current transporting part of the resistor. As already mentioned, microcracks are known to be mainly concentrated at the kerf tip. This explains the experimentally found difference in $\overline{\Delta R}^{\prime} / \bar{R}^{\prime}$ values $(2-4$ times higher for $D$-cut than for $L$-cut), and also explains the difference between $\overline{\Delta l}$ values for the two types of cut (see Figure 5.)

For both $D$-cut and $L$-cut a tendency for $\overline{\Delta l}$ values for short resistors to increase is observed. The cause for this is probably the increasing thickness of the resistor close to the terminals. ${ }^{4}$ As we can see in Figure 5. the $f$-value has only little effect on the

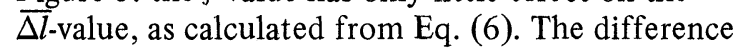
is generally within the standard deviation for $\Delta l$ and confirms again the theory of microcracking at the kerf tip as the predominant mechanism for long term drift in thick film resistors of high stability.

\section{OPTIMIZATION FOR LOW DRIFT}

Eq. (5) has now got all parameter values fixed, and it is thus possible to discuss the trim strategy for the high ohmic resistors we have chosen as an object for our study. Such a strategy must include the choice of cut type, $f$-value, $L$-value and $W$-value. It is also necessary to define the maximal drift, $\left(\overline{\Delta R}^{\prime} / \bar{R}^{\prime}\right) \max$, that can be accepted. It can be argued that the drift after trimming should not exceed two times the bulk drift $\Delta R_{\square} / R_{\square}$ of an untrimmed resistor. We therefore put $\left(\Delta R^{\prime} / R^{\prime}\right) \max =2 \Delta R_{\square} / R_{\square}$ and insert this value to the left of the equal sign in Figure 5.

Since the parameters $\lambda, \Delta \lambda, \Delta R_{\square} / R_{\square}$ and $\Delta l$ have already been determined, from Eq. (5) we obtain the allowed range of $f$-values corresponding to a certain $L^{\prime} / W$ value for resistors with PtAu terminals. For $D$-cut and $L$-cut two different $f-L^{\prime} / W$ zones have been calculated (See Figure 6). With the chosen widths of these resistors, we conclude that $L$-cut is the best choice for $L^{\prime} / W>2$. For shorter resistors a

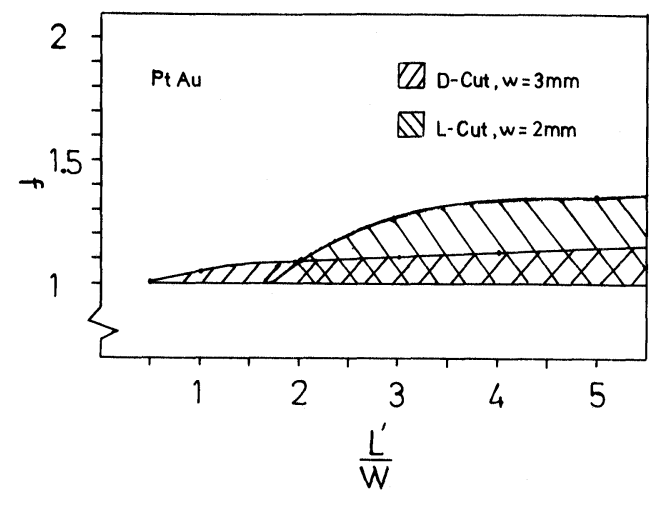

FIGURE 6 Allowed $f-L^{\prime} / W^{\prime}$ zones for $\left(\Delta R^{\prime} / R^{\prime}\right) \max =2$ $\Delta R_{\square} / R_{\square}$.

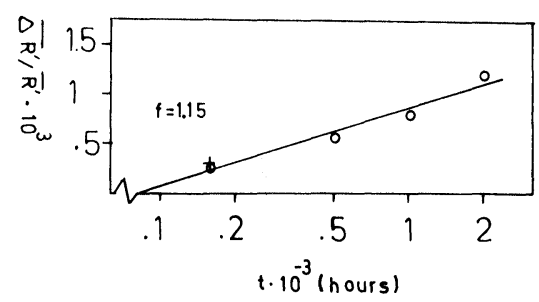

FIGURE 7 Drift in aged thickfilm resistors as a function of time in $150^{\circ} \mathrm{C}$. (o experimental, + theoretical).

$D$-cut with low trimming degree should be preferred due to the increase in $\Delta l$ for short $L$-cut resistors (see Figure 5).

In order to experimentally confirm the conclusions above, a series of 40 resistors ( $100 \mathrm{kohm} / \square)$ with $W=2 \mathrm{~mm}$ and $L / W=3$ were printed on PtAu terminals. Printing-firing parameters were the same as for the other resistors in this paper. $L$-cut trimming, was performed with the same laser parameters as before. The resistors were aged at $150^{\circ} \mathrm{C}$ for successively longer times and $\overline{\Delta R^{\prime}} \mid \bar{R}^{\prime}$ was plotted in Figure 7. The " + " in Figure 7 is the theoretically calculated drift at $168 \mathrm{~h}$ using the results from untrimmed resistors according to section 3 and with the $\overline{\Delta l}$-value equal to $0.25 \mu \mathrm{m}$ according to Eq. 6 (also see Figure 5).

\section{CONCLUSIONS}

A linear model for calculation of drift in untrimmed and trimmed thick film resistors has been developed. By aid of a numerically calculated $f^{-1}$ versus 
$l / W$-representation (for both $D$ - and $L$-cut), it has been possible to calculate the increase in trim cut depth $(\Delta l)$ after ageing.

From measurements on aged untrimmed resistors, it has been shown that $\overline{\Delta R} / \vec{R}$ is linearly dependent on $L^{\prime-1}$ according to Eq. (3). The $\lambda$-value used as fitting parameter was in good agreement with the EMPA and voltage probe measurements of Cattaneo et al. ${ }^{5}$

Trimmed resistors with varying geometries and with $f$-values situated between 1.4 and 3.3 have been aged. The corresponding, calculated $\overline{\Delta l}$ values were found to be unsensitive to the cut depth $(l)$, but sensitive to the length of the resistor. This is in good agreement with the microcracking model, ${ }^{2,3,4}$ and confirms that microcracking at the kerf tip is the dominant mechanism for trim induced drift at $150^{\circ} \mathrm{C}$.

A trim strategy for $D$-and $L$-cut was worked out, and the result is presented in the zone diagram in Figure 6 . The strategy was practically tested on a series of resistors with PtAu terminals. The low drift per time decade was in good agreement with the theoretical predictions and confirmed that high ohmic thick film resistors can be optimized for applications where high thermal stability is required.

\section{REFERENCES}

1. M. V. Coleman, Radio and Electron. Eng. 145, 3 (1975).

2. K. R. Bube, Am. Ceram. Soc. Bull. pp. 528-531, (May 1975).

3. R. E. Coté and R. C. Headley, Proc. Europ. Hybrid Microel. Conf. pp. XVI 1-8 (1977).

4. J. S. Shah, and L. Berrin, IEEE Trans. Comp. Hyb. and Manuf. Techn. Vol. CHMI 1, 2 pp. 130-135 (1978).

5. A. Cattaneo, M. Cocito, F. Forlani and M. Prudenziati, Electro-comp. Sci and Technol. 4 pp. 205-211 (1977).

6. J. Sundvall, Private communication.

7. E. J. Swensson, G. O. Vincent and J. C. Riley, Proc. Electron. Comp. Conf. (1972). 

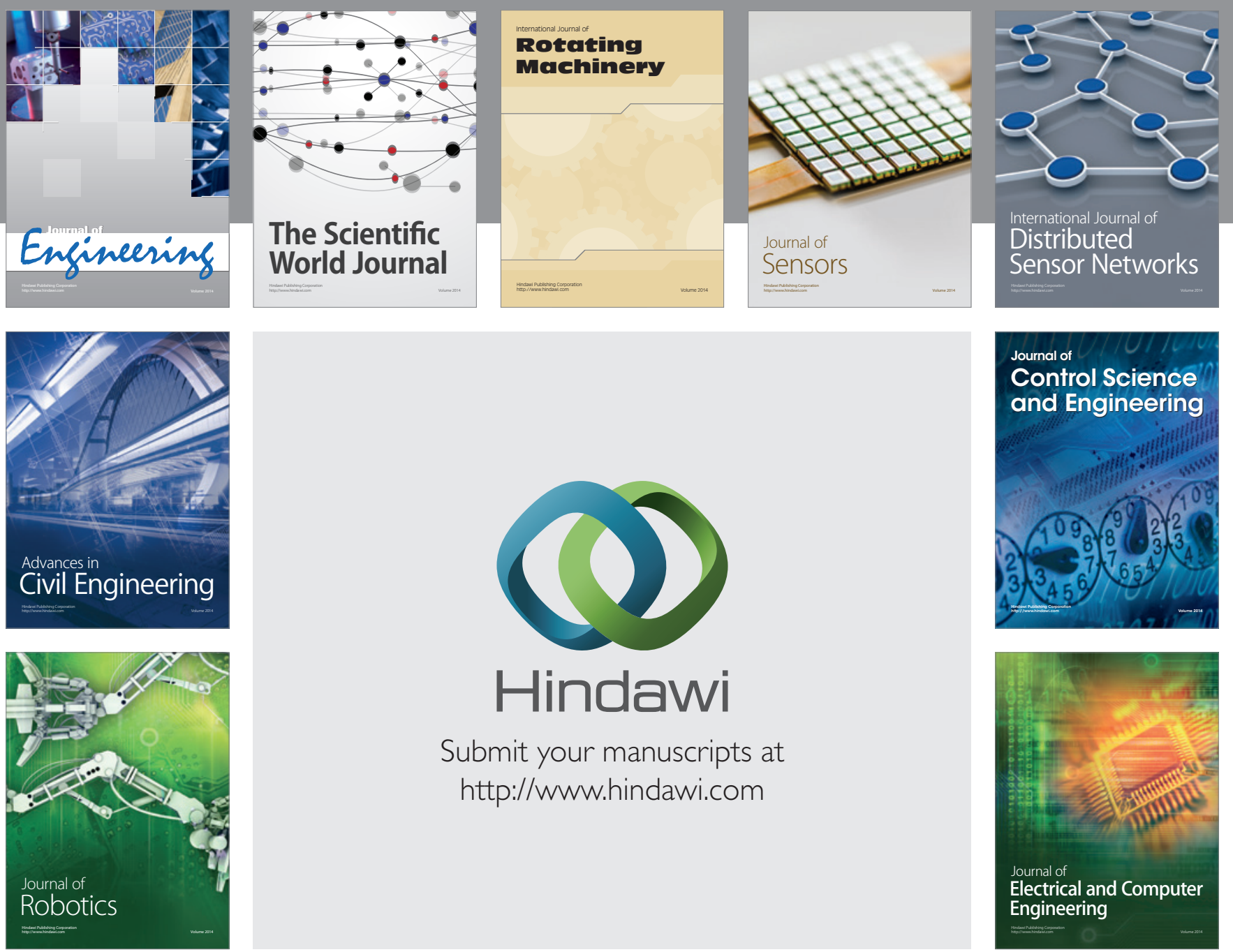

Submit your manuscripts at

http://www.hindawi.com
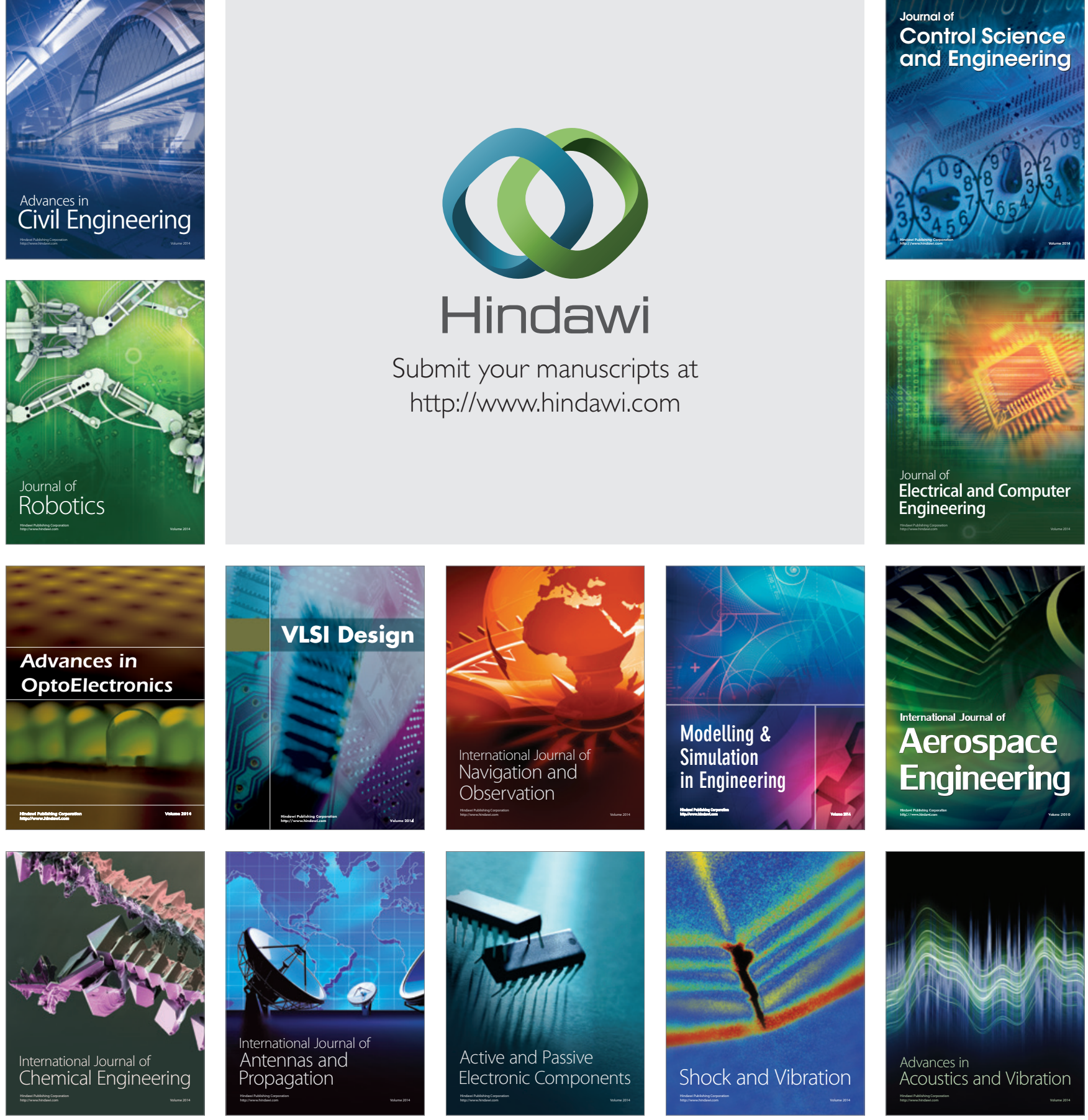